\title{
PowerScope: An Efficient Treatment Modality for Skeletal Class II Malocclusion
}

\author{
${ }^{1}$ Kimi Mittal, ${ }^{2}$ Kamal Bajaj, ${ }^{3}$ Manish Bansal, ${ }^{4}$ Rashmi Puri
}

\begin{abstract}
Skeletal class II malocclusion is one of the major complicated conditions to manage in orthodontics. Skeletal class II malocclusions due to lower jaw retrusion are usually treated by functional appliances during the peak of puberty. Due to the noncompliance with conventional myofunctional appliances, the evolution of fixed functional appliances took place. The present case report reports the ability of PowerScope in the correction of skeletal class II malocclusion due to lower jaw retrusion. This case report shows a patient aged 14 years who reported to the Department of Orthodontics and Dentofacial Orthopedics with a chief complaint of forwardly placed upper front teeth. In this case, McLaughlin, Bennett, Trevisi (MBT) 0.022" prescription was used to treat functional jaw retrusion initially, and then PowerScope was used. Cephalograms were obtained before treatment, during the middle stage of treatment, and after treatment. The results of cephalometric analysis showed considerable improvement in maxillomandibular relationship, facial profile, and appearance of patient. In this case, the chief component for correction of class II malocclusion was considerable displacement of mandible in the forward direction. Excellent results for management of class II malocclusion are provided by PowerScope, as such cases can be treated by a nonextraction approach if we use PowerScope as the treatment modality.
\end{abstract}

Keywords: Alignment and leveling, Class II division 1 malocclusion, Nonextraction, PowerScope.

How to cite this article: Mittal $\mathrm{K}$, Bajaj $\mathrm{K}$, Bansal M, Puri R. PowerScope: An Efficient Treatment Modality for Skeletal Class II Malocclusion. J Mahatma Gandhi Univ Med Sci Tech 2017;2(3):171-175.

Source of support: Nil

Conflict of interest: None

\section{INTRODUCTION}

Among the major challenges to orthodontists is class II malocclusion. ${ }^{1}$ Class II malocclusion can be of two types:

\footnotetext{
${ }^{1}$ Senior Lecturer, ${ }^{2}$ Professor and Head, ${ }^{3}$ Assistant Professor ${ }^{4}$ Resident (Third Year)

1,2,4 Department of Orthodontics and Dentofacial Orthopaedics Mahatma Gandhi Dental College and Hospital, Jaipur, Rajasthan India

${ }^{3}$ Department of Microbiology, Sawai Man Singh Medical College and Hospital, Jaipur, Rajasthan, India
}

Corresponding Author: Kimi Mittal, Senior Lecturer, Department of Orthodontics and Dentofacial Orthopaedics, Mahatma Gandhi Dental College and Hospital, Jaipur, e-mail: mittalkimimittal88@ gmail.com
Skeletal or dental. ${ }^{2}$ Skeletal class II malocclusion may be due to a protruded upper jaw or retruded lower jaw or a combination of both. Factors affecting the management of class II malocclusion include grade of the malocclusion and the remaining growth of the patient. As per McNamara, ${ }^{3}$ mostly the class II malocclusion is due to posterior positioning lower jaw, not due to protrusion of upper jaw. Treatment often recommended for class II malocclusion due to retrusion of lower jaw is functional appliance in order to advance the lower jaw. ${ }^{4-7}$ For patients who are in active growth period, removable functional appliances like FR, Clark's twin block, bionator, and activator can be used. ${ }^{8}$ If the patient comes after cessation of growth, fixed functional appliances, such as Forsus fatigue resistant device (FRD), Ritto appliance, Universal bite jumper, fixed twin block, Herbst, and Jasper jumper can be better alternatives in terms of the patient acquiescence.

PowerScope, a descendant of Herbst type II appliance, is in vogue in treatment of class II malocclusion. PowerScope was developed by Andy Hayes who worked in conjunction with American Orthodontics (Fig. 1). ${ }^{9}$ PowerScope grapples with vital requirements of orthodontists including simple insertion, large range of motion, patient's comfort, and much more. No studies have been reported yet on treatment outcomes of class II malocclusion with fixed orthodontic treatment combined with

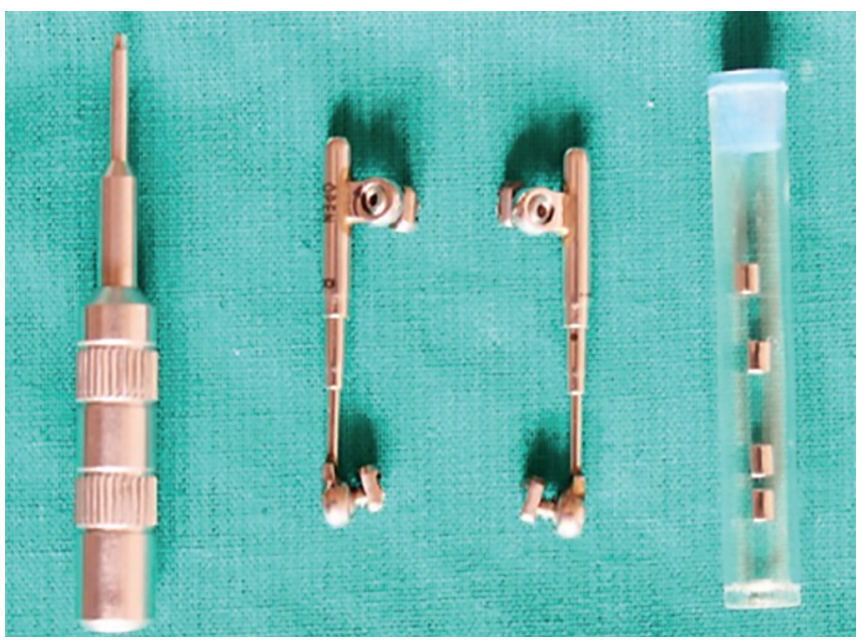

Fig. 1: PowerScope appliance armamentarium consisting of telescopic push rods (right and left), hexagonal screwdriver, and crimpable shims 
PowerScope. Treatment of skeletal class II malocclusion with nonextraction approach using PowerScope has been presented in this article.

The benefits of PowerScope are:

- Standard-sized one-piece appliance, fixed in upper and lower arches and suitable for all the patients

- Laboratory set-up is not required

- Wire-to-wire installation is swift and trouble-free

- Acquiescence at no cost

- Same force level is maintained throughout the treatment

- Need of headgear tube is not required

- It can be used either with banded or with bonded molar tube

- Debonding of buccal tube or canine bracket does not occur with the PowerScope assembly

- Comfortable to patient, as it is less bulky and esthetically pleasing

- Good oral hygiene can be maintained

\section{Appliance Design}

PowerScope is provided in a standard size suitable to all the patients. All the appliances are prefabricated with attachment nuts for swift and trouble-free on-chair application. ${ }^{9}$ The PowerScope appliance can be inserted to upper and lower archwire with the help of a nut and hexagonal screw. It provides a ball and socket joint. The appliance consists of a telescopic mechanism made up with inner shaft, middle, and outer tubing; between middle tubing and outer tubing, there is a nickel-titanium (NiTi) spring that provides constant force of $260 \mathrm{~g} .{ }^{9}$

\section{Appliance Insertion}

The PowerScope appliance is inserted mesially to the upper first molar and distally to the lower cuspid with the help of its nut and hexagonal screw over the highest possible rectangular SS wire.

\section{Appliance Activation}

An activation dot is marked over the push rod of the appliance (left and right) which helps us in knowing whether the appliance is activated or not. If the dot mark is seen, it means that the appliance does not exert any kind of force and it is inactive, and to reactivate the appliance, crimpable shims of different dimensions of 2 or $3 \mathrm{~mm}$, as per the requirement, are attached to the shaft.

\section{Case Study}

A 14-year-old female patient presented with a chief complaint of upper front teeth that were forwardly placed. Clinical examination revealed protruded upper lip, incompetent lips, recessive chin button, and convex

profile with posterior divergence (Fig. 2). The diagnosis was class II division 1 malocclusion with an overbite of $6 \mathrm{~mm}$ and an overjet of $9 \mathrm{~mm}$ (Fig. 3).

Cephalometric analysis revealed orthognathic maxilla, and retrognathic mandible with A point, nasion, and B point (ANB) angle of $6^{\circ}$ (Fig. 4 and Table 1). Dental readings suggested protruded upper anterior teeth with mildly retruded mandibular incisors. The mandibular plane angle was within normal range, indicating an average growth pattern. Hand-wrist radiographs suggested that the patient was in active growth phase. Pretreatment orthopantomogram (OPG) showed erupting third molars in first, second, and third quadrants (Fig. 5).

\section{TREATMENT OBJECTIVES}

- To obtain asymmetrical balanced class I buccal occlusion ruling out tooth exfoliation

- To obtain good facial esthetic by lengthening of mandible during its peak growth

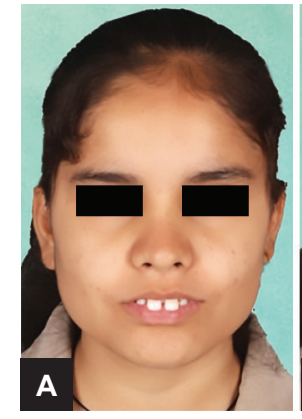

Figs 2A to C: Extraoral pretreatment photos. (A) Frontal; (B) smiling; (C) left lateral
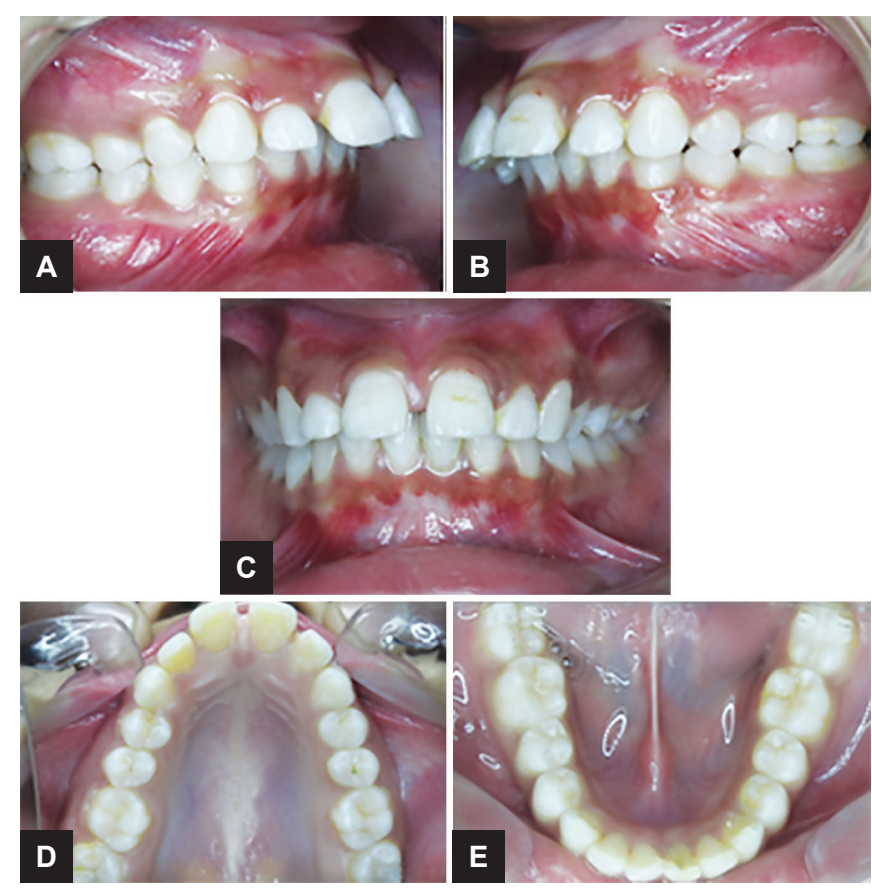

Figs $3 \mathrm{~A}$ to $\mathrm{E}$ : Intraoral pretreatment photos. (A) Right lateral; (B) left lateral; (C) frontal; (D) lower occlusal; (E) upper occlusal 
PowerScope: An Efficient Treatment Modality for Skeletal Class II Malocclusion

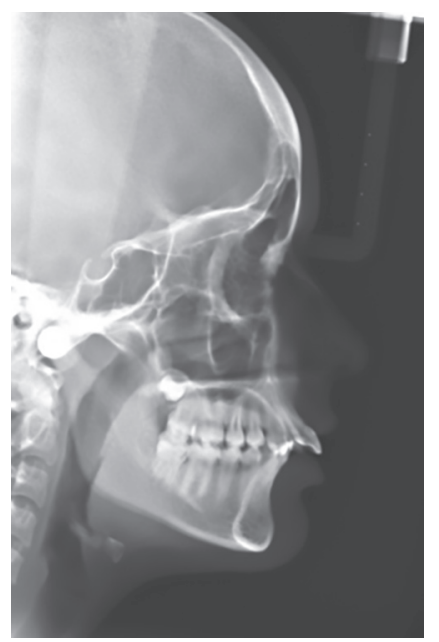

Fig. 4: Pretreatment lateral cephalogram

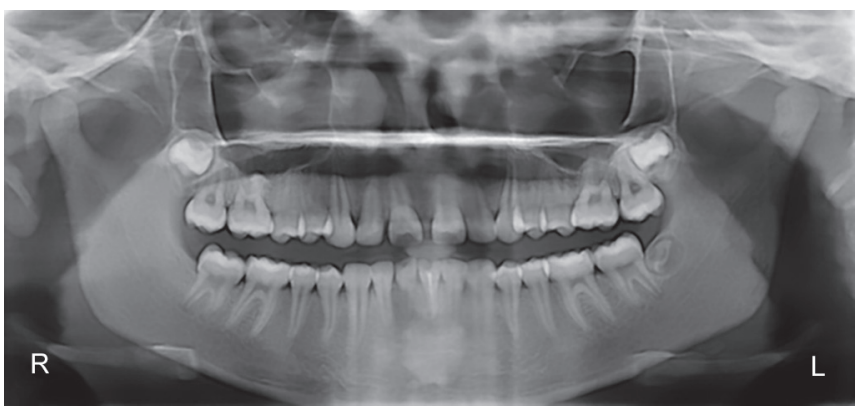

Fig. 5: Pretreatment OPG

- Restrict maxillary growth in sagittal and vertical plane

- Avoid any undue backward rotation of the mandible

\section{TREATMENT PLAN}

Treatment plan included nonextraction approach, using MBT 0.022" slot preadjusted appliance. After alignment
Table 1: Pretreatment and posttreatment skeletal, dental, and soft tissue changes observed in lateral cephalogram

\begin{tabular}{lll}
\hline Measurements & Pretreatment & Posttreatment \\
\hline Position of maxilla & & \\
SNA & $82^{\circ}$ & $82^{\circ}$ \\
Na perp. to pt A & $2 \mathrm{~mm}$ & $1 \mathrm{~mm}$ \\
Position of mandible & & \\
SNB & $76^{\circ}$ & $79^{\circ}$ \\
N-Pog (facial angle) & $87^{\circ}$ & $89^{\circ}$ \\
Maxillomand relation & & \\
ANB & $6^{\circ}$ & $3^{\circ}$ \\
Witt's & $3 \mathrm{~mm}$ & $1 \mathrm{~mm}$ \\
AB to N-Pog & $-18^{\circ}$ & $-9^{\circ}$ \\
Beta angle & $24^{\circ}$ & $28^{\circ}$ \\
Dental & & \\
Interincisal angle & $105^{\circ}$ & $120^{\circ}$ \\
Upper incisor to SN & $103^{\circ}$ & \\
\hline
\end{tabular}

and leveling, the appliance chosen to bring the mandible forward into class I relationship was PowerScope, followed by finishing and detailing.

\section{TREATMENT PROGRESS}

Initially, 0.014" NiTi wire was ligated in both arches to align and level the teeth. Alignment and leveling were accomplished in a duration of 9 months. After alignment and leveling, $0.019^{\prime \prime} \times 0.025^{\prime \prime}$ stainless steel wire was ligated in both the upper and lower arches. Then, PowerScope was placed for advancement of lower jaw (Fig. 6). To avert the undue proclination of mandibular anteriors, a $5^{\circ}$ labial root torque was given in lower archwire. The patient's profile was improved considerably after forward placement of lower jaw.
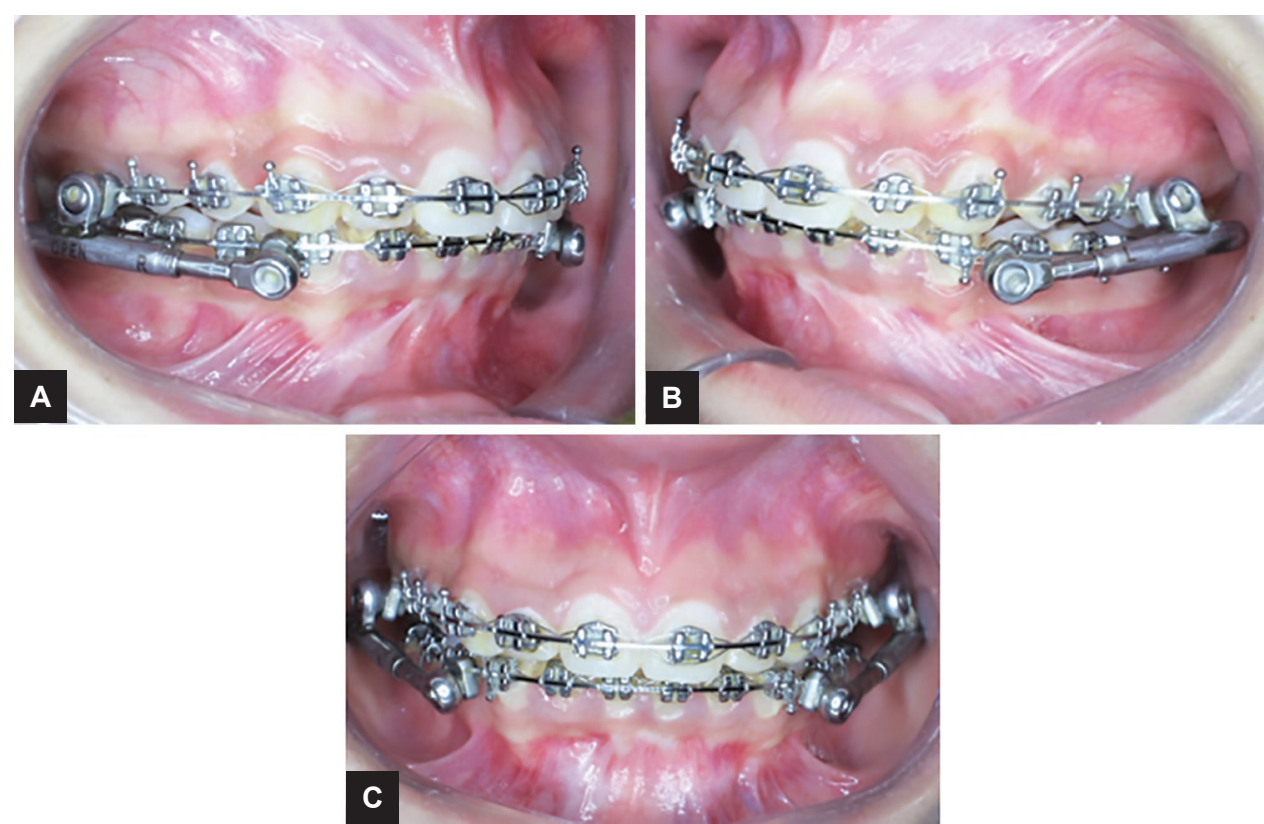

Figs 6A to C: Mandibular advancement by using power scope 


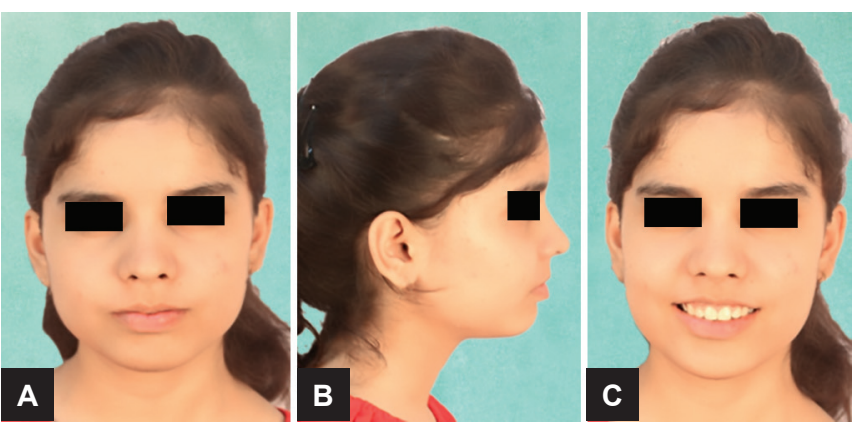

Figs 7A to C: Extraoral posttreatment photos. (A) Frontal; (B) right lateral; (C) smiling

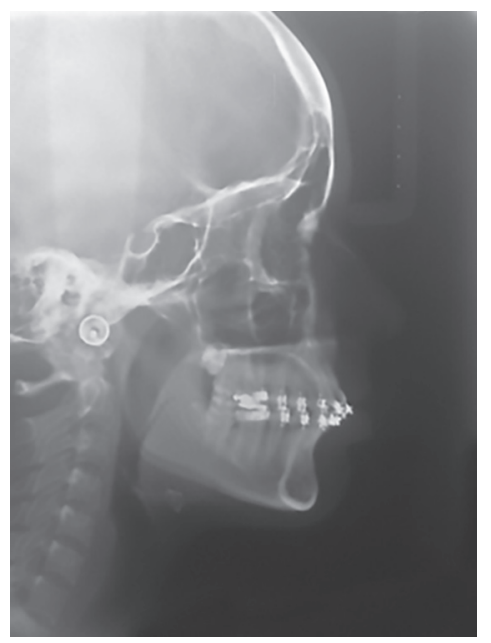

Fig. 9: Posttreatment lateral cephalogram

\section{TREATMENT OUTCOME}

Pretreatment and posttreatmentcephalometric outcomes were compared, which revealed substantial enhancement in soft tissue (Fig. 7), dental (Fig. 8), skeletal (Fig. 9), and posttreatment parameters using PowerScope appliance (Table 1). The posttreatment cephalometric measurements indicated beneficial anteroposterior skeletal changes. The SNA angle $\left(82^{\circ}\right)$ was not changed during treatment. Due to forward placement of lower jaw, the SNB angle changed from 76 to $79^{\circ}$. Thus, ANB angle was reduced by $3^{\circ}$. On Wit's appraisal, $2 \mathrm{~mm}$ advancement of $\mathrm{BO}$ was noticed. Beta angle increased by $4^{\circ}$ (from 24 to $28^{\circ}$ ).

Angulation of upper incisors decreased from 122 to $103^{\circ}$. Patient's profile was improved from convex to straight. The relationship of lower lip to E line improved from $-2 \mathrm{~mm}$ to $-1 \mathrm{~mm}$, indicating forward placement of lower lip. Nasolabial angle was significantly improved. Posttreatment OPG showed root parallelism (Fig. 10). The treatment could consequently achieve a harmonious face with an attractive smile which could be verified by the superimposition of hard and soft tissues (Fig. 11). Patient and clinician were satisfied with these results.
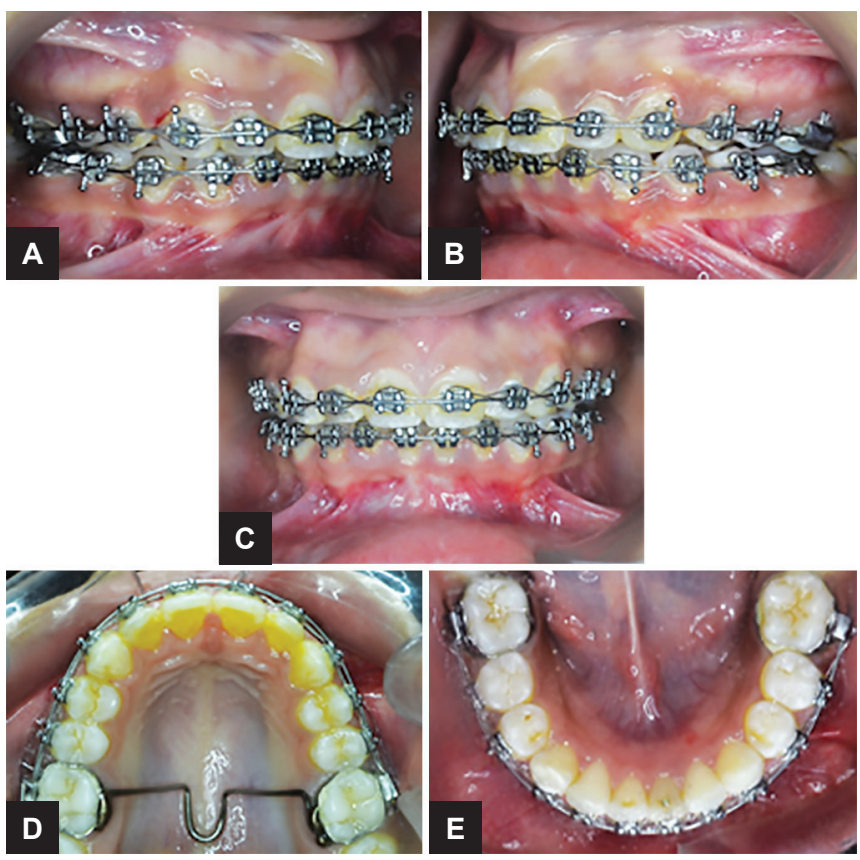

Figs $8 \mathrm{~A}$ to $\mathrm{E}$ : Intraoral posttreatment photos

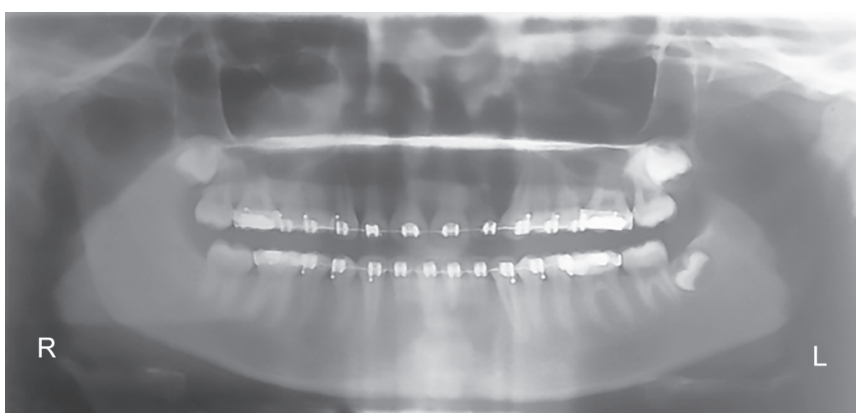

Fig. 10: Posttreatment OPG

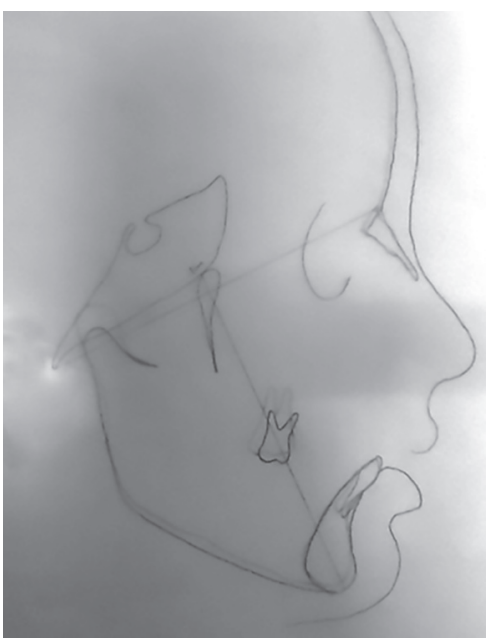

Fig. 11: Superimposition shows growth at condyles

\section{DISCUSSION}

One of the persistent challenges to orthodontists in all malocclusion is class II malocclusion. ${ }^{10}$ For correction of skeletal class II malocclusion, various appliances and treatment modalities have been attempted. Skeletal class II 
malocclusions due to shortening of lower jaw are often treated with some appliances, which can modify the growth of mandible, called as functional appliances. An orthopedic force at mandibular condyle is created by a functional appliance. ${ }^{11}$ Functional appliances can be of two types-fixed or removable appliances. American Orthodontics recently added PowerScope in the category of fixed functional appliance. Many studies have been done on various fixed functional appliances, such as Herbst, Jasper jumper, Eureka Spring, Universal bite jumper, and Forsus FRD, but no reports are presently available concerning PowerScope. ${ }^{12-14}$ Treatment of the case, discussed here, is done by PowerScope, taking into account the advantages of PowerScope over the other conventional appliances. The PowerScope can be used in all class II patients. It is a standard-sized single-piece appliance, which can be fixed on upper and lower arches. Due to appliance being unisized, there is no need of size selection, and thus, it is time saving to order the appliance. Crimpable shims are provided with the PowerScope to customize it. This article elucidates the changes in skeletal, dental, and soft tissue parameters after the treatment is completed with PowerScope, a fixed appliance.

\section{CONCLUSION}

The correction of class II malocclusion due to posterior positioning of lower jaw with extraction of teeth in upper arches can provide a deleterious result in facial esthetics. So, these kinds of patients should be treated using class II correctors, such as PowerScope. It is one of the best treatment alternatives for class II correction, as it improves the esthetic appearance of the patient by forward displacement of lower jaw, ensuring outstanding and everlasting stable outcomes.

\section{REFERENCES}

1. Graber, TM.; Rakosi, T.; Petrovic, A. Dentofacial orthopedics with functional appliances. St. Louis (MO): C. V. Mosby Co.; 1997. pp. 346-352.
2. Proffit, WR. Malocclusion and dentofacial deformity in contemporary society. In: Profit WR, Fields HW, Sarver DM, editors. Contemporary orthodontics. 4th ed. St. Louis (MO): Mosby Elsevier; 2007. pp. 3-23.

3. McNamara JA Jr. Components of class II malocclusion in children 8-10 years of age. Angle Orthod 1981 Jul;51(3): 177-202.

4. Nelson C, Harkness M, Herbison P. Mandibular changes during functional appliance treatment. Am J Orthod Dentofacial Orthop 1993 Aug;104(2):153-161.

5. Patel HP, Moseley HC, Noar JH. Cephalometric determinants of successful functional appliance therapy. Angle Orthod 2002 Oct;72(5):410-417.

6. Cozza P, Baccetti T, Franchi L, De Toffol L, McNamara JA Jr. Mandibular changes produced by functional appliances in class II malocclusion: a systematic review. Am J Orthod Dentofacial Orthop 2006 May;129(5):599.e1-599.e12.

7. Schaefer AT, McNamara JA Jr, Franchi L, Baccetti T. A cephalometric comparison of treatment with the Twin-block and stainless steel crown Herbst appliances followed by fixed appliance therapy. Am J Orthod Dentofacial Orthop 2004 Jul;126(1):7-15.

8. Ritto AK, Ferreira AP. Fixed functional appliances-a classification. Funct Orthod 2000 Spring;17(2):12-30, 32.

9. Cetlin NM, Ten Hoeve A. Nonextraction treatment. J Clin Orthod 1983 Jun;17(6):396413.

10. Pancherz H, Ruf S, Kohlhas P. "Effective condylar growth" and chin position changes in Herbst treatment: a cephalometric roentgenographic long-term study. Am J Orthod Dentofacial Orthop 1998 Oct;114(4):437-446.

11. Nelson B, Hansen K, Hägg U. Class II correction in patients treated with class II elastics and with fixed functional appliances: a comparative study. Am J Orthod Dentofacial Orthop 2000 Aug;118(2):142-149.

12. Heinig N, Göz G. Clinical application and effects of the Forsus spring. A study of a new Herbst hybrid. J Orofac Orthop 2001 Nov;62(6):436-450.

13. Vogt W. The Forsus fatigue resistant device. J Clin Orthod 2006 Jun;40(6):368-377.

14. El-Sheikh MM, Godfrey K, Manosudprasit M, Viwattanatipa N. Force-deflection characteristics of the fatigue-resistant device spring: an in vitro study. World J Orthod 2007 Spring;8(1): 30-36. 